\title{
Integrating a Model of Analytical Quality Assurance into the V-Modell XT
}

\author{
Stefan Wagner and Michael Meisinger \\ Institut für Informatik \\ Technische Universität München \\ Boltzmannstr. 3, D-85748 Garching b. München, Germany \\ \{wagnerst,meisinge\}@in.tum.de
}

\begin{abstract}
Economic models of quality assurance can be an important tool for decision-makers in software development projects. They enable to base quality assurance planning on economical factors of the product and the used defect-detection techniques. A variety of such models has been proposed but many are too abstract to be used in practice. Furthermore, even the more concrete models lack an integration with existing software development process models to increase their applicability. This paper describes an integration of a thorough stochastic model of the economics of analytical quality assurance with the systems development process model VModell XT. The integration is done in a modular way by providing a new process module - a concept directly available in the V-Modell XT for extension purposes - related to analytical quality assurance. In particular, we describe the work products, roles, and activities defined in our new process module and their effects on existing V-Modell XT elements.
\end{abstract}

\section{Categories and Subject Descriptors}

D.2.9 [Software Engineering]: Management; D.2.8 [Software Engineering]: Metrics; D.2.5 [Software Engineering]: Testing and Debugging

\section{General Terms}

Economics, Verification, Reliability

\section{Keywords}

Software quality economics, quality costs, quality model, quality assurance, process model, V-Modell

\section{INTRODUCTION}

Software quality costs and economics have been subject to research for decades now. Consequently, there is a variety of corresponding models on all levels of abstraction

Permission to make digital or hard copies of all or part of this work for personal or classroom use is granted without fee provided that copies are not made or distributed for profit or commercial advantage and that copies bear this notice and the full citation on the first page. To copy otherwise, to republish, to post on servers or to redistribute to lists, requires prior specific permission and/or a fee.

SOQUA'06, November 6, 2006, Portland, OR, USA

Copyright 2006 ACM 1-59593-584-3/06/0011 ...\$5.00. as a result of this research. The development and improvement of these models is important, especially for the decision makers in real software projects 17 . This becomes obvious when considering that there are many estimates that assign $30-50 \%$ of the development costs to quality assurance 14 9. A newer study of the National Institute of Standards and Technology of the United States 19 found that even $80 \%$ of the development costs are caused by the detection and removal of defects. Hence, models are needed to control and minimise these costs. Yet, for this to be feasible we need to incorporate them into existing development processes. Thereby, we make them operational and accessible for decision-makers.

\subsection{Problem}

Most models are not directly applicable in a real development process. They often only classify the relevant costs but do not show how to use this classification. Even operational models often neglect the fact that they need to be used in the context of a specific process model. The usage of such models is mainly in an ad-hoc manner and they are not systematically included in process models.

\subsection{Contribution}

The contribution lies in the seamless integration of a model for analytical software quality assurance into the existing process model V-Modell XT. We show how our QA model is operationally used and which roles, products, and activities are involved in using the model in practice. This allows an easy adoption of the model for a project that follows the V-Modell XT. Although similar ad-hoc usages of such models are practice in some companies, we are not aware of an earlier systematic integration.

\subsection{Outline}

First, we introduce quality economics in general and in terms of the analytical model in Sec.2. In Sec.3 3 we describe the basics of the considered process model and its underlying meta-model. Sec. 4 then shows the integration of the model with the V-Modell XT. We finish with related work in Sec.6 and final conclusions in Sec. 7

\section{QUALITY ECONOMICS}

We first describe the cost types and other factors that are important in the context of the economics of analytical quality assurance. Then we give a short overview of the analytical model from 24, 25. that is to be integrated in the process model V-Modell XT later. 


\subsection{Cost Types and Factors}

We reduce the classical PAF (Prevention, Appraisal, Failure) model of quality costs to an AF (Appraisal, Failure) model. We ignore prevention costs that contain the costs of preventing defects by constructive QA because constructive QA has significantly different characteristics. Appraisal costs contain all costs for checking artefacts to detect defects, e.g., test specification and execution. The debugging is then part of the failure costs. When the failure occurs inhouse it incurs internal failure costs. Failures during operation at the customer cause external failure costs. We refine these parts so that we can identify the relevant cost factors. The complete refined model is shown in Fig. 1 The appraisal costs are detailed to setup and execution costs. The former constituting all initial costs for buying test tools, configuring the test environment, and so on. The latter includes costs that are connected to actual test executions or review meetings, mainly personnel costs.

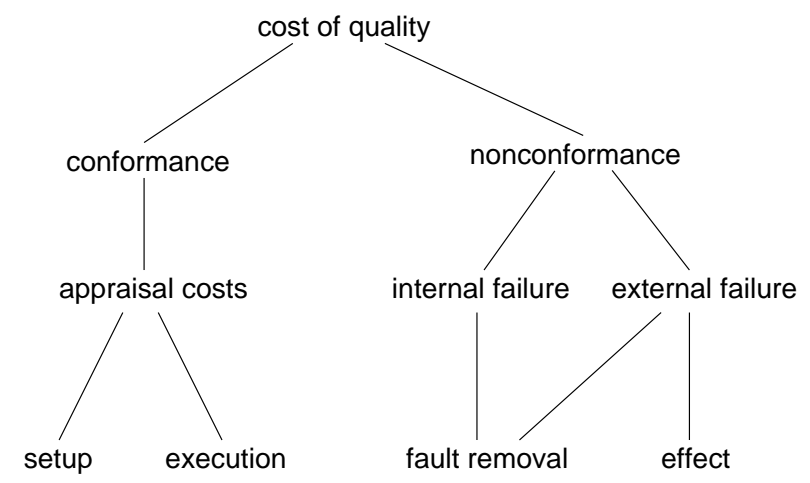

Figure 1: The refined cost types

On the nonconformance side, we have fault removal costs that can be attributed to the internal failure costs as well as the external failure costs. This is because if we found a fault and wanted to remove it, it would always result in costs no matter whether caused by an internal or external failure. Actually, there does not have to be a failure at all. Considering code inspections, faults are found and removed that have never caused a failure during testing. Fault removal costs also contain the costs for necessary re-testing and re-inspections.

External failures also cause effect costs. Those are all further costs associated with the failure apart from the removal costs. For example, compensation costs could be part of the effect costs, if the failure caused some kind of damage at the customer site. We might also include other costs such as loss of sales because of bad reputation in the effect costs.

Furthermore, there are also technical factors that are important for the quality economics of analytical quality assurance. The two main factors that we consider in the following model are (1) the difficulty of defect-detection and (2) the failure probability of faults. We denote the probability that a specific defect-detection technique does not detect a defect of a specific type as its difficulty. This factor has been shown to be influential 24]. A smaller but still substantial impact stems from the failure probability of faults. This is important because many faults occur only with a very small probability during operation 2 .

\subsection{Analytical Model}

We use the stochastic quality assurance model from 24 , 25. as the model to be integrated in the V-Modell XT. Actually, we only consider the practical model from this work because that is the one to be applied. It is derived from a theoretical model that incorporates more factors and more detail. However, the main factors described above are still contained in this model.

The main idea of the model is to compute the expected values of the costs and benefits of quality assurance. For this purpose, they are structured in three components: direct costs, future costs and revenues. The determination of the expected values is based on average values calculated from literature and finished projects. During the project, measured data can be used to refine the results.

We define $\tau_{i}$ to be the defect type of fault $i$. It is determined using the defect type distribution of older projects. In this way we do not have to look at individual faults but analyse and measure defect types for which the determination of interesting quantities is possible during quality assurance. We will not further elaborate the concept of defect types but refer to defect classification approaches from IBM 10] or HP [8]. For the sake of a simple presentation, we first give equations for a single defect-detection technique and generalise that to a combination of techniques.

\subsubsection{Single Economics}

We start with the direct $\operatorname{costs} d_{A}$ of a defect-detection technique. They are all costs that occur directly by using the technique.

$$
E\left[d_{A}\left(t_{A}\right)\right]=u_{A}+e_{A}\left(t_{A}\right)+\sum_{i}\left(1-\theta_{A}\left(\tau_{i}, t_{A}\right)\right) v_{A}\left(\tau_{i}\right)
$$

where $u_{A}$ is the average setup cost for technique $A, e_{A}\left(t_{A}\right)$ is the average execution cost for $A$ with effort $t$, and $v_{A}\left(\tau_{i}\right)$ is the average removal cost for defect type $\tau_{i}$.

The future costs $o_{A}$ are those costs that will occur when defects are not detect by the technique.

$$
E\left[o_{A}\left(t_{A}\right)\right]=\sum_{i} \pi_{\tau_{i}} \theta_{A}\left(\tau_{i}, t_{A}\right)\left(v_{F}\left(\tau_{i}\right)+f_{F}\left(\tau_{i}\right)\right) .
$$

Finally, the revenues $r_{A}$ are the saved future costs, i.e., the costs that will not incur because the technique finds them.

$$
E\left[r_{A}\left(t_{A}\right)\right]=\sum_{i} \pi_{\tau_{i}}\left(1-\theta_{A}\left(\tau_{i}, t_{A}\right)\right)\left(v_{F}\left(\tau_{i}\right)+f_{F}\left(\tau_{i}\right)\right),
$$

where $f_{F}\left(\tau_{i}\right)$ is the average effect costs of a fault of type $\tau_{i}$.

\subsubsection{Combined Economics}

The extension to more than one technique needs to consider whether the defects have been found by earlier used techniques. The following is the equation for the expected value of the direct costs:

$$
\begin{gathered}
E\left[d_{X}\left(t_{X}\right)\right]=\sum_{x \in X}\left[u_{x}+e_{x}\left(t_{x}\right)+\sum_{i}\left[\left(1-\theta_{x}\left(\tau_{i}, t_{x}\right)\right)\right.\right. \\
\left.\left.\cdot \prod_{y<x}\left(\theta_{y}\left(\tau_{i}, t_{y}\right)\right) v_{x}\left(\tau_{i}\right)\right]\right],
\end{gathered}
$$

where $X$ is the ordered set of the used defect-detection techniques. Also the expected value of the combined future costs 
$o_{X}$ can be formulated in the practical model using defect types.

$$
\begin{array}{r}
E\left[o_{X}\left(t_{X}\right)\right]=\sum_{i}\left[\pi_{\tau_{i}} \prod_{x \in X}\left(\theta_{x}\left(\tau_{i}, t_{x}\right)\right)\right. \\
\left.\cdot\left(v_{F}\left(\tau_{i}\right)+f_{F}\left(\tau_{i}\right)\right)\right]
\end{array}
$$

Finally, the expected value of the combined revenues $r_{X}$ are defined accordingly.

$$
\begin{gathered}
E\left[r_{X}\left(t_{X}\right)\right]=\sum_{x \in X} \sum_{i}\left[\pi_{\tau_{i}}\left(1-\theta_{x}\left(\tau_{i}, t_{x}\right)\right) \prod_{y<x}\left(\theta_{y}\left(\tau_{i}, t_{y}\right)\right)\right. \\
\left.\cdot\left(v_{F}\left(\tau_{i}\right)+f_{F}\left(\tau_{i}\right)\right)\right]
\end{gathered}
$$

\subsubsection{Needed Quantities}

Using the practical model, we identify only seven different types of quantities that are needed to use the model:

- Estimated number of faults: $I$

- Distribution of defect types

- Difficulty functions for each technique and type $\theta_{x}\left(\tau_{i}\right)$

- Average removal costs for type $\tau_{i}$ with technique $x$ : $v_{x}(i)$

- Average removal costs for type $\tau_{i}$ in the field: $v_{F}(i)$

- Average effect costs for type $\tau_{i}$ in the field: $f_{F}(i)$

- Failure probability of fault of type $\tau_{i}: \pi_{\tau_{i}}$

For an early application of the model, average values from a literature review can be used as first estimates. We did an extensive analysis of those values in 23] and ranked them using sensitivity analysis in 24. For more specific estimations we can use more sophisticated methods: The COQUALMO model 6] allows to determine an estimate of the number of faults contained in the software. The defect removal effort for different defect types can be predicted using an association mining approach of Song et al. 21.

\subsubsection{Optimisation}

Optimisation is the key to using the model operationally in a project. It allows to calculate an optimal effort distribution for the used defect-detection techniques. Only two of the three components of the model are needed because the future costs and the revenues are dependent on each other. There is a specific number of faults that have associated costs when they occur in the field. These costs are divided in the two parts that are associated with the revenues and the future costs, respectively. The total always stays the same, only the size of the parts varies depending on the defect-detection technique. Therefore, we use only the direct costs and the revenues for optimisation and consider the future costs to be dependent on the revenues.

Hence, the optimisation problem can be stated by: maximise $r_{X}-d_{X}$. By using Eq. 4 and Eq. 6] we get the following term to be maximised.

$$
\begin{gathered}
\sum_{x}\left[-u_{x}-e_{x}\left(t_{x}\right)+\sum_{i} \sum_{c}\left(q_{i}(c)\left(1-\theta_{x}^{t_{x}}\right) .\right.\right. \\
\left.\left.\prod_{y<x}\left(\theta_{y}(c)^{t_{y}}\right)\left(\pi_{i} v_{F}\left(\tau_{i}\right)+\pi_{i} f_{F}\left(\tau_{i}\right)-v_{x}\left(\tau_{i}\right)\right)\right)\right]
\end{gathered}
$$

For the optimisation purposes, we usually also have some restrictions, for example a maximum effort $t_{\max }$ with $\sum_{x} t_{x} \leq$ $t_{\text {max }}$, either fixed length or none $t_{A}=\{0,100\}$, or some fixed orderings of techniques, that have to be taken into account. The latter is typically true for different forms of testing as system tests are always later in the development than unit tests. We assume there is sufficient tool-support available to solve this optimisation problem

\section{V-MODELL XT}

The V-Modell XT 4, 18, is a recently released German software and system development standard. It covers all relevant management, engineering and supporting processes of software development, for instance project management, quality assurance, offer, bidding and contract management, and also technical disciplines such as requirements engineering, system design and integration, software development and more specific engineering activities.

The goals of the V-Modell XT are to provide a generic development process model, which is easy to understand and to use, flexibly adaptable to the needs of organisations and projects, and reproducibly leading to developed products of higher quality with less cost and resources spent.

\subsection{Concepts of the V-Modell XT}

In order to extend the V-Modell XT it is imperative to know its main concepts. The V-Modell XT is based on a rigorous meta-model, which defines all concepts and their relationships. The entire process model strictly follows this meta-model, which is a prerequisite for flexible extensibility. The main concepts of the V-Modell XT are:

- Work Products are the main project results and artefacts (documents, models, code, deliverable systems). They have a defined structure and prescribed content, and can be structured further into specific subjects (sub-sections). Work Products have a responsible creator and will be quality checked. An evaluation specification defines the requirements for their quality.

- Product Dependencies define the consistency relations between the contents of different work products. Adhering to and checking product dependencies makes sure that all work products in a project will be created and kept consistent to existing products. Product dependencies are an important means to assure overall product quality and to trace information across products, for instance from requirements to software architecture elements.

- Activities define the actions that need to be performed in order to create the work products. Activities can be structured further into sub-activities. Activities provide support for the actual doing within a project. There is exactly one activity per work product. In case there are multiple instances or iterations of a certain 
work product, the respective activity is performed several times, accordingly. Each activity creates a work product; therefore it is directly followed by a product evaluation for QA, if required.

- Roles describe profiles of responsibility for the people working in the project. Roles will be impersonated by specific people in the project.

- Process Modules group together Work Products, Activities and Roles, as well as other V-Modell XT elements into self-contained units covering certain project processes, such as project management, requirements management, systems integration, software development, etc. Process modules have a hierarchy of dependence. They can be understood, applied and modified independently if adhering to these dependencies. Process modules are the main units of tailoring and extension of the V-Modell XT. Important for the VModell XT extension mechanism is that process modules can define work products, subjects, activities, subactivities etc. that modularly and seamlessly extend existing processes of the core process modules.

- Tailoring is the process of adapting the V-Modell XT to a specific project or organisation. Tailoring consists of selecting the appropriate process modules out of the repository of available ones. After the tailoring, a consistent and adapted software development process exists. It is indistinguishable to the user from which process modules which specific elements - work products, their subjects, extensions etc - came from.

\subsection{V-Modell XT Quality Assurance Mecha- nisms}

Quality assurance is a cornerstone within a process model such as the V-Modell XT. It is the main means to ensure result quality by constructive and analytical means. The QA mechanisms of the V-Modell XT are manifold and cover the following areas:

1. Organisational Quality Management. Organisational units define general quality standards, guidelines and metrics to be applied in projects. They also archive results in metric catalogues to be used by subsequent projects in order to continuously improve the process on the organisational level. The V-Modell XT does not define these processes but provides an interface to them. The responsible role is the Quality Manager.

2. Project Setup. During project setup, the Project Manual and the QA Manual are created which define standards, general rules and guidelines that must be applied during the project. The QA Manual in particular defines the constructive and analytical methods that are applied for each different type of project result to ensure the desired product quality. Responsible for these tasks are the Project Leader and the $Q A$ Manager.

3. Product Evaluation. Project results are evaluated according to a product specific evaluation specification, following an evaluation procedure. The means applied for evaluating a certain product vary depending on its importance and criticality. $Q A$ Reports are created in defined intervals describing the state and potential quality problems of the product under construction based on the results of the product evaluations. Evaluators are responsible for evaluations and the $Q A$ Manager for the reports.

4. Project Management. The project management decides about general project progress based on project and $Q A$ Reports. In case of need, it applies corrective means to increase product quality. Project management reports to the customer and higher levels in the organisation; it is responsible for the overall project result quality as well as for observing agreed upon delivery deadlines and costs. The responsible role is the Project Leader.

\subsection{Existing Analytical Process}

The V-Modell XT already contains an optional process module named "Measurement and Analysis". It is very lightweight and only provides the ideas of applying quality analysing metrics within a project. This process module depends only on the core process module of "Project Management". Besides, it does not have any other dependencies, and does not extend the general process module "Quality Assurance".

In order to keep the compliance level with the standard V-Modell XT as high as possible, we replace the existing "Measurement and Analysis" process module with our own one. However, we reuse the elements defined in the existing process module and complement them with new elements and extended content.

\section{INTEGRATION}

In this section we show the exemplary embedding of our model in the process model $V$-Modell XT [4], as described in the previous section. An integration with other process models such as the RUP 11] can be done accordingly. For the V-Modell XT integration, we define a new process module "Measurement and Analysis of Analytical QA" containing roles, products, and activities. We first give a brief overview of the general analytical QA process and then describe the contents of the process module in more detail.

\subsection{Overview}

The diagrams shown in Fig. 2 and Fig. 3 give an overview of the usage of our model as part of the V-Modell XT. We relate the activities to the different QA processes of the $\mathrm{V}$ Modell XT described in Sec. 3.2.

1. The Quality Manager is responsible for defining and documenting cross-project quality standards, metrics, and methods, within the Quality Management Manual. Furthermore, he defines and maintains the Metrics Catalogue that must contain all the necessary input factors summarised in Sec. 2.2.3 The Quality Manager must also provide an infrastructure that is able to store the corresponding metrics.

2. Within a project, the Project Leader is responsible for setting up the project initially. The Project Leader performs basic estimations for the project including the defect estimate needed for our model. He can get supporting data from similar projects using the Metrics Infrastructure. Based on these estimates, he 


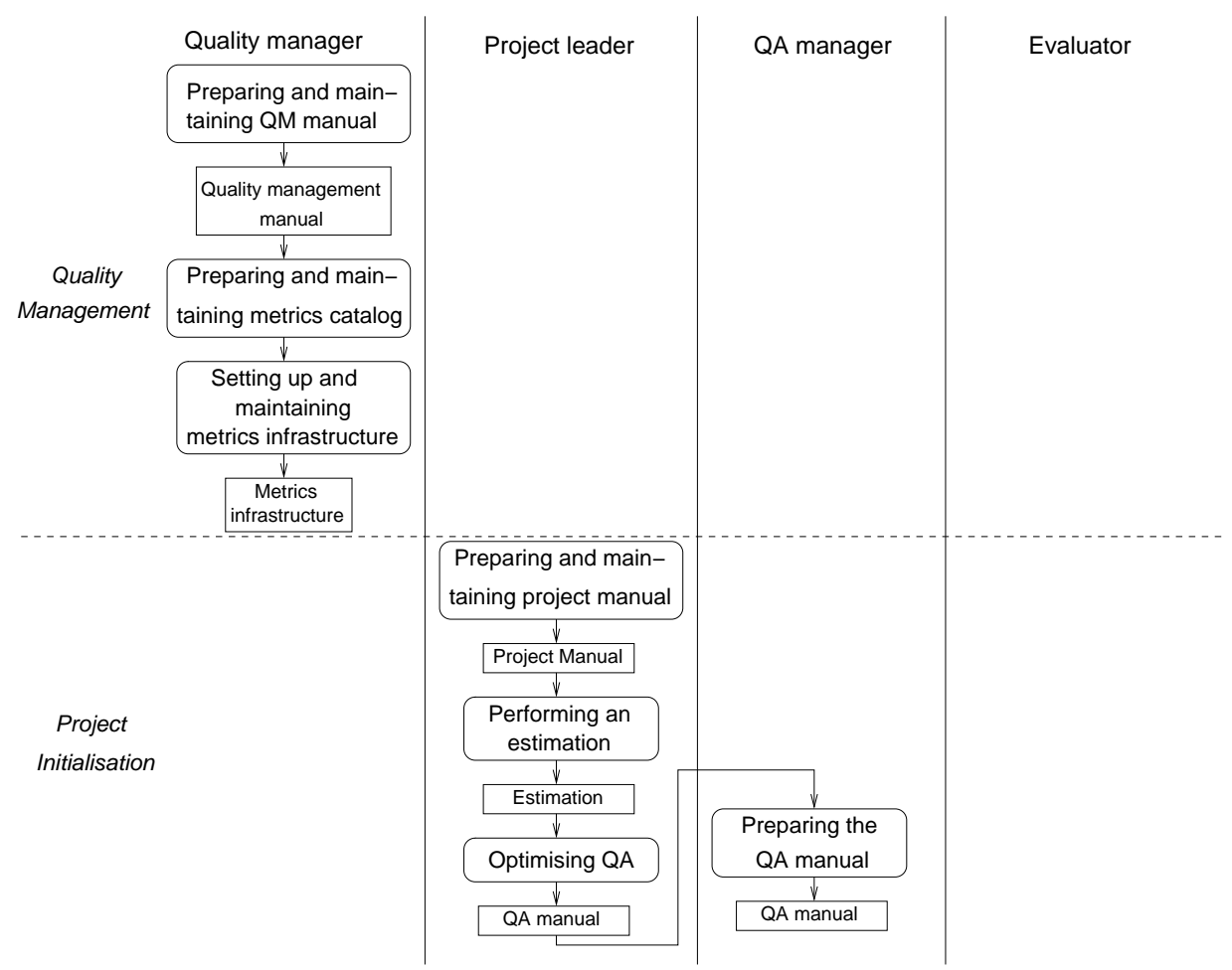

Figure 2: An abstract activity diagram of the model application (first part)

uses our model to calculate an optimised quality assurance and he documents this as part of the $Q A$ Manual. Also during project setup, the $Q A$ Manager is responsible for implementing the guidelines from the Quality Management Manual within the project. In collaboration with the Project Leader he defines the $Q A$ manual and provides input for the QA relevant sections of the Project Manual. The project setup results in a completed Project Manual where project goals and guidelines for supporting project processes, such as measurement and analysis are defined.

3. During the course of the project, Evaluators are performing the product QA tasks. Depending on the specific requirements for specific work products as expressed in the $Q A$ Manual, they are preparing Evaluation Specifications and Evaluation Procedures for each evaluated product. The Project Plan, under responsibility of project management, plans the occurrences of the product evaluations and QA measures. According to these guidelines, the Evaluators perform the actual product evaluations and document the results in Evaluation Reports, which include all measurements that can be later used in our model such as the number and type of the detected defects. In regular intervals, the QA Manager compiles Quality Status Reports out of the product Evaluation Reports.

4. The Project Leader and project management are continuously assessing project progress and are responsible for making regular project progress decisions, which act as "quality gates". One important source of input for these decisions are the Quality Status Reports.
Project management can use the available information to apply our model to evaluate different scenarios and optimise the further QA strategy. The results will refine the Project Plan and might lead to an update of the $Q A$ Manual. When the project is finished, the project leader collects the Measurement Data relevant for our model and forwards it to the Quality Manager who stores it in the metrics infrastructure.

\subsection{Extensions to the V-Modell XT}

In the following we show in greater detail the extensions to the V-Modell XT we made to embed our analytical QA model. The V-Modell XT was created as a generic process model which already puts significant effort on QA and related management processes. It thus already provides the basic framework for analytical QA. To fit our model, we have to extend existing elements of the $\mathrm{V}$-Modell and add a few new ones. Mainly this is only more detail on the explicit measurement and collection of the data for the model input factors. For our extension, we concentrate on products, activities and roles.

The integration of our analytical quality model affects the responsibilities of 4 roles that already exist in the $\mathrm{V}$ Modell XT. Their role profile descriptions are sufficiently abstract and fit our purposes. Thus, only slight extensions need to be performed:

- The Quality Manager is responsible for quality assurance standards across all projects and for an efficient and effective quality management system. In particular, he develops a systematic quality management and creates and maintains the Quality Management Manual. Most importantly in our context, he defines rules 


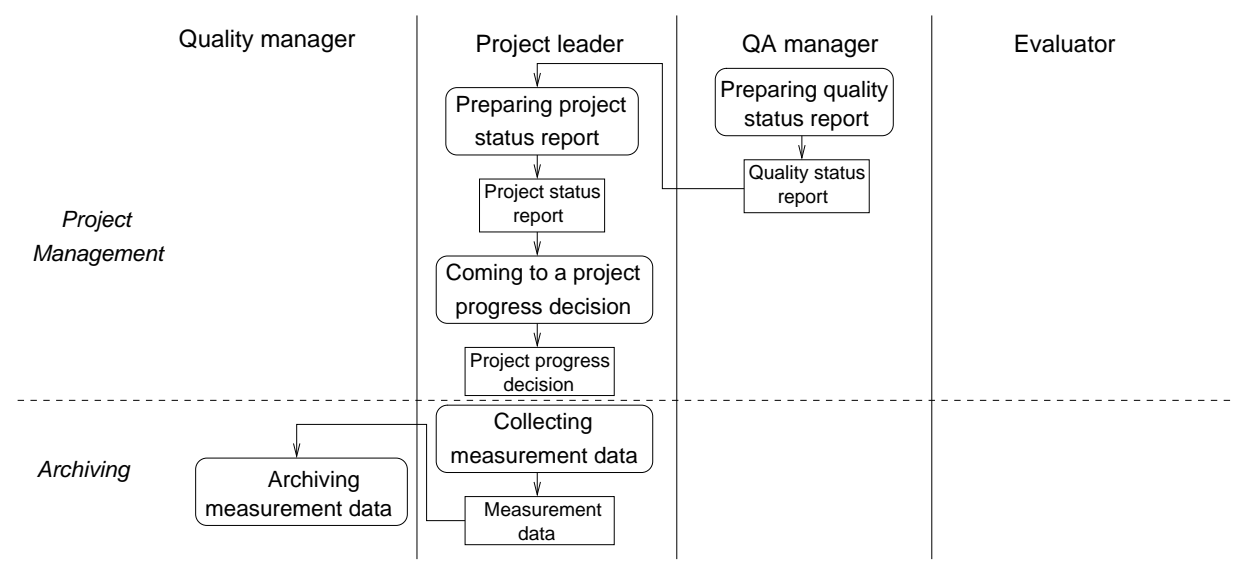

Figure 3: An abstract activity diagram of the model application (second part)

and approaches how projects plan and perform quality assurance techniques. Furthermore, he defines which QA techniques should be used in general and helps in choosing appropriate techniques for a specific project. We add that he is responsible for setting up and maintaining the Metrics Infrastructure.

- The Project Leader, as the leader of the project's execution, plans and controls the project's progress. In particular, he makes the basic estimates for project planning and decides on future changes based on status reports. The main extension for this role is that he uses our model for optimising the resource distribution for quality assurance and also collects the necessary measurement data for our model.

- The $Q A$ Manager controls the quality in a project and thereby supervises all quality assurance. He is responsible for the Quality Status Reports and also plans the QA work in collaboration with others. There is only the small addition that in his Quality Status Report the necessary measurements for the model must be contained.

- The Evaluator - also called inspector although he not only uses inspections - creates evaluation specifications and using those evaluates the artefacts created in the project. Hence, he uses defect-detection techniques, e.g., reviews and tests, on those artefacts and reports the results. Also for the Evaluator it is necessary that he documents the necessary measurements for the model factors.

Work products are the main V-Modell elements and also the core project results. Work products have one responsible role. The following list shows the work products that need to be considered, extended or added to apply our model:

- The Quality Management Manual is a work product that we add to capture - among other subjects - organisation-wide definitions of the metrics that need to be collected for the usage of the model. Thus Metrics Definitions is one subject in this product. Responsible role for this product is the Quality Manager. The VModell XT mentions such a document but does not officially introduce it.
- We also add the Metrics Catalog, which exists in the V-Modell XT only as subject of an organisation-wide process model, for process adaptation and improvement (ORG) projects. We reuse the subject description and establish it as a full product under the responsibility of the Quality Manager. We additionally explicitly require the incorporation of the factors from Sec. 2.2.3 Thereby, we can reference this product in regular development projects.

- The Metrics Infrastructure is the third new work product under the responsibility of the Quality Manager. It is in essence similar to the existing Project Management Infrastructure but not project specific. In our context it needs to store the measured data for the relevant metrics of our model and provide access to it across projects over an extended period of time.

- We extend the existing product Estimation with a new subject Estimation of the defect content that contains data, which we use later in our model. Responsible is the Project Leader.

- We extend the existing products Evaluation Report and Quality Status Report with new subjects that will contain the necessary measurement data for the factors of Sec. 2.2.3 Responsible are Evaluator resp. $Q A$ Manager roles.

- We use the existing work product Measurement Data to capture all data that is collected in the course of the project for calculating the relevant metrics of our model. Responsible for this product is the Project Leader.

- The Metrics Analysis is another existing work product under the responsibility of the Project Leader. It contains detailed analyses of the relevant metrics of our model based on the previously measured data.

Each work product has exactly one associated activity. During execution of such an activity one instance or iteration of the work product is created or edited. After the completion of the activity, a product evaluation is performed according the QA guidelines. The following list explains 
the relevant activities and sub-activities to apply our model. Most are related to the given work products above:

- We introduce a new activity Preparing and maintaining Quality Management Manual doing what its name says. A sub-activity describes how the necessary metrics for applying our model are selected and defined.

- We introduce the existing sub-activity Preparing and maintaining metrics catalogue of the V-Modell XT as full activity creating and maintaining the product Metrics Catalog.

- The new activity Setting up and maintaining the Metrics Infrastructure will make sure that a data repository is available for the measurement data.

- The main element of our analytical quality assurance model is introduced into the V-Model XT as new subactivity Optimise $Q A$. It belongs to the activity Preparing the $Q A$ Manual. This sub-activity is performed by the Project Leader with help from the $Q A$ Manager based on his estimates and data from similar projects. He calibrates the model and optimises it (w.r.t. cost or ROI) so that an optimal resource distribution is found. This is then documented in the QA Manual.

- The activity Collecting Measurement Data describes how the resp. product is created and edited. A new sub-activity activity Archiving Measurement Data requires that the measurement data will be stored during the project and at its end so that they are available across projects and for new projects.

- The existing activity Calculating and Analysing Metrics is extended with a new sub-activity to extract the data that is to be stored for future projects in the Metrics Infrastructure.

- We add to the activity Coming to a Project Progress Decision a new sub-activity which uses our economics model as basis for the decision. Different scenarios can be analysed and an optimal effort (or resource) distribution can be calculated.

We package all above described new work products and activities as well as product/activity extensions in form of subjects and sub-activities as part of our new process module. We add general descriptions and an overview of the process module contents. Thereby we have performed a fully modular extension of the V-Model XT that a Project Leader can choose or not choose to apply during initial V-Modell tailoring for a new project.

\section{DISCUSSION}

In summary, we find that our model blends well with the V-Modell XT. The necessary changes and additions to use the model fit with the existing structure and require only additions and slight extensions of existing V-Modell XT elements. They all can be packages nicely as a process module. An embedding into other process models with a similar structuring should be possible with a comparable effort.

One of the challenges of integrating our analytical quality assurance model into the V-Modell XT is the scope of the described process. Our model covers activities on both the organisational level - spanning multiple projects - and the project level. The main focus of the V-Modell XT is to describe a process for conducting a particular project: once a project is initiated and the decision to use the V-Modell XT is made, the tailoring activity will result in a project specific process. The V-Modell XT does not specifically cover organisation-wide processes, such as quality management and continuous process improvement. However, this is not a limitation, because it can easily be extended - we have done this for our integration. This results in a responsibility of the organisation to apply the V-Modell XT process across projects and to provide the necessary infrastructure.

A consequence of our modular extension of the V-Modell is the sometimes artificial separation between project specific and organisation specific work product (and activity) definitions, such as the Project Infrastructure and the Metrics Infrastructure. Additionally, the existing V-Modell XT process support for the Introduction and Maintenance of Organisation-Specific Process Models - the process module ORG - covers part of the organisation-wide activities without being combinable and integrated with project specific processes. Thus, we chose to introduce some redundancy for the sake of modularity and clear understandability.

One of the prerequisites of our approach is the necessity to have comparable projects which yield expressive metrics data. The higher the degree of similarity between the projects and the better the measured metrics data, the more precise will our model be able to optimise the QA processes.

\section{RELATED WORK}

General efficiency models of defect-detection techniques such as the inspection model of Kusumoto et al. 12 or the testing model of Morasca and Serra-Capizzano [13] are aiming at analysing specific techniques and their application. However, they are typically not usable for planning purposes in a software project. Cost models based on reliability models, e.g., Pham [15], aim to decide when to stop testing. However, they are only applicable to the system testing phase.

More economic-oriented models such as iDAVE Boehm et al. 3] or the model of Slaughter, Harter, and Krishnan 20. are typically more abstract or coarse-grained than the used model. Moreover, the question when and how to use those models is not completely clear. Especially, we are not aware of an integration into an existing process model.

Punter et al. 16] aim also at a practical application of product evaluation with specific goals. However, they concentrate more on the actual evaluation process using the ISO 14598 standard which we assume given by the V-Modell XT. They also do not explicitly discuss the aim of using the evaluation results for future optimisations.

Cai et al. 5] propose a method of optimal and adaptive testing with cost constraints. They discuss that it is effective to adapt testing and to explore the interplay between software and control. However, their model does only consider testing and is not explicitly integrated in a complete process model.

Ambler uses process patterns in [1] to describe task-specific self-contained pieces of processes and workflows in a reusable way. Such patterns can be applied to solve complex tasks when needed. Störrle 22] shows how process patterns can be described in great detail using UML. The idea of process patterns is further refined by Gnatz et al. 7] in form of a 
modular and extensible software development process based on collections of independent process components. These process patterns essentially are the basis of the extension mechanism of the V-Modell XT.

\section{CONCLUSIONS}

Analytical models of quality assurance would be a valuable tool for project managers and other decision-makers in software projects. There is a variety of such models available on different levels of abstraction. However, the adoption in practice is still weak. One main problem is that the usage of those model is often not clear. Especially, when and how the model should be used in an existing process model is typically not specified by the model proposers.

In this paper, we show the exemplary integration of a detailed model of analytical quality assurance in the process model V-Modell XT. We are not aware of other models of quality assurance that have explicitly been integrated into an existing process model. The benefits of this work are twofold: (1) organisations that follow already the V-Modell XT have now simple means to also incorporate the analytical model into their process. (2) It has been shown that such an integration can be done relatively simple and with little effort. Therefore, this should be also possible with other process models and hence the usage of models of QA can be increased.

For future work, we consider tool support as the other important aspect of pushing the use of such models in software organisations. Hence, we plan to build an easy to use tool implementation that helps in applying the model. It is also to investigate whether our claim w.r.t. the easy integration into other process models really holds.

\section{ACKNOWLEDGMENTS}

We are grateful to Ulrike Hammerschall for the discussion on a first integration.

\section{REFERENCES}

[1] S. W. Ambler. More Process Patterns: Delivering Large-Scale Systems Using Object Technology. Cambridge University Press, 1999.

[2] B. Boehm and V. R. Basili. Software Defect Reduction Top 10 List. IEEE Computer, 34(1):135-137, 2001.

[3] B. Boehm, L. Huang, A. Jain, and R. Madachy. The ROI of Software Dependability: The iDAVE Model. IEEE Software, 21(3):54-61, 2004.

[4] Bundesrepublik Deutschland. V-Modell XT, 1.2 edition, 2006. http://www.v-modell-xt.de/.

[5] K.-Y. Cai, Y.-C. Li, W.-Y. Ning, W. E. Wong, and H. Hu. Optimal and Adaptive Testing with Cost Constraints. In Proc. 2006 International Workshop on Automation of Software Test (AST '06), pages 71-77. ACM Press, 2006.

[6] S. Chulani and B. Boehm. Modeling Software Defect Introduction and Removal: COQUALMO (COnstructive QUALity MOdel). Technical Report USC-CSE-99-510, University of Southern California, Center for Software Engineering, 1999.

[7] M. Gnatz, F. Marschall, G. Popp, A. Rausch, and W. Schwerin. The living software development process. Software Quality Professional, 5(3), June 2003.
[8] R. B. Grady. Practical Software Metrics for Project Management and Process Improvement. Prentice-Hall, 1992.

[9] C. Jones. Applied Software Measurement: Assuring Productivity and Quality. McGraw-Hill, 1991.

[10] S. H. Kan. Metrics and Models in Software Quality Engineering. Addison-Wesley, 2nd edition, 2002.

[11] P. Kruchten. The Rational Unified Process. An Introduction. Addison-Wesley, 2nd edition, 2000.

[12] S. Kusumoto, K. ichi Matasumoto, T. Kikuno, and K. Torii. A New Metric for Cost Effectiveness of Software Reviews. IEICE Transactions on Information and Systems, E75-D(5):674-680, 1992.

[13] S. Morasca and S. Serra-Capizzano. On the Analytical Comparison of Testing Techniques. In Proc. 2004 ACM SIGSOFT International Symposium on Software Testing and Analysis (ISSTA '04), pages 154-164. ACM Press, 2004.

[14] G. J. Myers. The Art of Software Testing. John Wiley \& Sons, 1979.

[15] H. Pham. Software Reliability. Springer, 2000.

[16] T. Punter, R. Kusters, J. Trienekens, T. Bemelmans, and A. Brombacher. The W-Process for Software Product Evaluation: A Method for Goal-Oriented Implementation of the ISO 14598 Standard. Software Quality Journal, 12(2):137-158, 2004.

[17] A. Rai, H. Song, and M. Troutt. Software Quality Assurance: An Analytical Survey and Research Prioritization. Journal of Systems and Software, 40:67-83, 1998.

[18] A. Rausch and M. Broy. Das V-Modell XT. Grundlagen, Erfahrungen und Werkzeuge. dpunkt.verlag, 2006. In German.

[19] RTI. The Economic Impacts of Inadequate Infrastructure for Software Testing. Planning Report 02-3, National Institute of Standards \& Technology, 2002 .

[20] S. A. Slaughter, D. E. Harter, and M. S. Krishnan. Evaluating the Cost of Software Quality. Communications of the ACM, 41(8):67-73, 1998.

[21] Q. Song, M. Sheperd, M. Cartwright, and C. Mair. Software Defect Association Mining and Defect Correction Effort Prediction. IEEE Transactions on Software Engineering, 32(2):69-82, 2006.

[22] H. Störrle. Describing Process Patterns with UML. In Proc. 8th European Workshop on Software Process Technology (EWSPT '01), pages 173-182. Springer, 2001.

[23] S. Wagner. A Literature Survey of the Quality Economics of Defect-Detection Techniques. In Proc. 5th ACM-IEEE International Symposium on Empirical Software Engineering (ISESE '06). ACM Press, 2006.

[24] S. Wagner. A Model and Sensitivity Analysis of the Quality Economics of Defect-Detection Techniques. In Proc. International Symposium on Software Testing and Analysis (ISSTA '06), pages 73-83. ACM Press, 2006.

[25] S. Wagner. Cost-Optimisation of Analytical Software Quality Assurance. PhD Dissertation, Technische Universität München, 2006. To appear. 